\title{
Efficacy and cost-effectiveness of a blended cognitive behavioral therapy for depression in Spanish primary health care: study protocol for a randomised non-inferiority trial
}

Ma Dolores Vara', Rocío Herrero ${ }^{2 *}$, Ernestina Etchemendy ${ }^{3}$, Macarena Espinoza ${ }^{1}$, Rosa Ma Baños ${ }^{1,4}$, Azucena García-Palacios ${ }^{2,4}$, Guillem Lera ${ }^{5}$, Blanca Folch ${ }^{5}$, Vicente Palop-Larrea ${ }^{5}$, Pilar Vázquez ${ }^{6}$, Manuel Franco-Martín ${ }^{6}$, Annet Kleiboer ${ }^{7}$, Heleen Riper ${ }^{7,8}$ and Cristina Botella ${ }^{2,4}$

\begin{abstract}
Background: Data from primary health care in Spain show a high prevalence of the major depressive disorder. Blended treatment (combination of face-to-face and online components) seems to be a very promising tool for the optimization and dissemination of psychological treatments in a cost-effective form. Although there is growing data that confirm the advantages of blended therapies, few studies have analyzed their application in regular clinical practice. The objective of the present paper is to describe the protocol for a clinical study aimed at exploring the clinical and cost-effectiveness of a blended cognitive behavioral therapy (b-CBT) for depression, compared to treatment as usual (TAU) in a primary health care setting.

Methods: A two-arm randomised controlled non-inferiority trial will be carried out, with repeated measures (baseline, 3 months, 6 months, and 12 months) under two conditions: b-CBT and TAU. The b-CBT program will consist in three face-to-face sessions and eight online sessions. The TAU is defined as the routine care delivered by the general practitioner for the treatment of depression in primary care. The primary outcome is a symptomatic change of depressive symptoms on the patient-health questionnaire (PHQ-9). Other secondary outcomes will be considered (e.g., quality of life, treatment preference). All participants must be 18 years of age or older and meet the diagnostic criteria for major depressive disorder according to the Diagnostic and Statistical Manual of Mental disorders 4th edition. 156 participants will be recruited (78 per arm).
\end{abstract}

Discussion: It is expected that b-CBT is clinically non-inferior when compared to TAU. This is the first study in Spain to use a b-CBT format in primary and specialized care, and this format could be an efficacious and cost-effective therapeutic strategy for the treatment of depression.

Trial registration: ClinicalTrials.gov NCT02361684. Registered on 8 January 2015. Currently recruiting participants.

Keywords: Blended treatment, Cognitive behavioral therapy, Depression, Internet-based treatment, Primary health care, Randomised non-inferiority trial

\footnotetext{
*Correspondence: rherrero@uji.es

2Department of Basic Psychology, Clinical and Psychobiology, Jaume I

University, Castellón, Spain

Full list of author information is available at the end of the article
} 


\section{Background}

Depression is a highly prevalent disorder with considerable personal and social costs in terms of quality of life, wellbeing, and economic effects [1-4]. In this regard, the World Health Organization (WHO) predicts that depression will be one of the three leading causes of the burden of disease by 2030 [5, 6]. Even though there are effective treatments for this emotional disorder (pharmacotherapy, psychotherapy or both) [7-9], these treatments fail to reach the growing number of people who need them [10]. Data from primary health care in Spain reveal a high prevalence of mental disorders (with depression at the top) [11-13] and an important gap between the number of people suffering from depression and the rate of treatment received $[14,15]$. Therefore, it becomes necessary to incorporate new approaches into the traditional ways of providing psychological treatments, in order to effectively respond to this need [10, 16]. Internet-based treatments are a cost-effective alternative that can improve treatment dissemination, becoming a useful resource to address the gap between treatment demand and supply. In recent years, several studies have assessed the effectiveness and acceptability of computer-based treatments for depression, obtaining positive results [17-19]. Regarding efficacy, several systematic reviews have found that online treatments are more effective when guided (ranging from automated reminders to encouragement and feedback by email, text messages, or brief telephone calls) [20, 21], highlighting the positive association between therapist contact and improvement rates [22]. Some data also show that guided self-help and face-to-face treatments do not significantly differ in their effectiveness [23-25].

Due to the benefits of including therapist support in Internet-delivered treatments, and seeking the best approach in terms of cost-efficacy, the "blended treatment" format emerges as a good alternative to address the growing need for access to evidence-based psychological treatments for mental disorders. Blended treatment usually refers to the combination of face-to-face and online components [26]. This format combines the best qualities of each therapeutic approach: broader dissemination, less therapist time, lower health services costs, and direct therapist-patient support and guidance, which may lead to greater adherence. There are previous experiences with this treatment format, with positive outcomes in terms of efficacy, adherence, and relapse prevention [27-31].

Despite the above, very little research has focused on blended treatments in regular clinical practice (e.g., [32]). If the goal is to diminish the burden of mental disorders and decrease the gap between treatment demand and true access to psychological support, it is necessary to assess the efficacy, feasibility, and cost-effectiveness of these types of treatments. Therefore, the aim of the present paper is to describe the protocol for a randomised controlled non-inferiority trial that compares the clinical and cost-effectiveness of blended cognitive behavioral therapy (b-CBT) for adults suffering from major depressive disorder (MDD) and treatment as usual (TAU) in a primary health care setting.

\section{Methods}

\section{Aim}

The aim of the study is to explore the clinical and costeffectiveness of a b-CBT for depression, compared to TAU, in primary care in Spain. This trial is part of the ECOMPARED project [European 7FP, $\mathrm{N}^{\circ}$ Agreement; 603,098], which includes several randomised controlled non-inferiority trials in both primary care and specialized care in eight European countries, in an effort to obtain clinically significant data.

The hypothesis is that b-CBT will be at least as effective as the TAU condition, defined as the routine care that patients receive in primary care in Spain when diagnosed with depression.

\section{Study design}

A two-arm randomised controlled non-inferiority and cost-effectiveness trial will be carried out, with repeated measures (baseline, 3 months, 6 months, and 12 months) and two conditions: b-CBT and TAU. The study will be conducted following the CONSORT statement (Consolidated Standards of Reporting Trials, http://www.consort-statement.org) [33, 34], the CONSORT-EHEALTH guidelines [35], and the Recommendations for Interventional Trials (SPIRIT) [36, 37].

\section{Sample size}

The sample size calculation is based on the noninferiority design and calculated for the primary clinical outcome: depression symptoms (3 months after baseline). Following Cohen [38], $\mathrm{f}=0.20$ represents an effect size of small magnitude, which is a conservative estimate of the subjective minimal important difference noted by patients. Taking into account the E-COMPARED project as a whole, and considering an alpha of 0.05 and a statistical power of 0.90 , the total sample size required to warrant these conditions is 1052 participants. Based on the literature on Internet-based treatments, a 30\% dropout rate is expected [39]. Thus, the total number of participants to be recruited will be 1200, 150 patients in each country involved in the project. However, in the case of the trial in Spain, we intend to include 156 participants (78 per arm). This sample size was calculated assuming that there is no difference between standard and blended depression treatment, considering a statistical power of 0.80 , and that the lower limit of a one-sided $95 \%$ 
confidence interval will be above a non-inferiority limit of - 0.4. A margin of 0.4 was judged acceptable, as this range of a small to moderate difference in effect size will not result in clinically important differences.

\section{Study population, recruitment, and eligibility criteria}

Participants who report depression to their general practitioner (GP) will be consecutively invited to participate in the trial. This recruitment will be implemented in primary care centers belonging to the Hospital of the Rivera in Valencia, and the Provincial Hospital of Zamora. All interested participants must give written informed consent to take part in the trial. To confirm inclusion/ exclusion criteria (see Table 1), a telephone screening interview will be carried out by a psychologist from the University of Valencia and the University of Castellón, using the MINI International Neuropsychiatric Interview for diagnosis version 5 (M.I.N.I. 5.0) [40-42]. If the participant meets the eligibility criteria, after completing the baseline measurement, randomization will be conducted.

An independent researcher from VU University in Amsterdam will create an allocation scheme using a block randomization design (variable block sizes) with a computerized random number generator (Random Allocation Software) at an allocation ratio of 1:1. Participants will be assigned to two conditions: b-CBT or TAU. The condition to which they are assigned will be communicated to the participants by telephone. Blinding for the treatment is not possible because it will be clear to both therapists and patients whether the treatment is blended or not. The follow-up measurements will be administered online and by telephone ( 3 months, 6 months, and 12 months). Figure 1 shows the study flow chart.

\section{Ethics}

The study follows the guidelines of the Helsinki Declaration [43]. All the researchers will follow the guidelines for Good Clinical Practice [44]. As noted, all the participants will be volunteers, and they will not receive any compensation for their participation. They will sign the informed consent once the study and its conditions have been explained. Participants will be able to withdraw

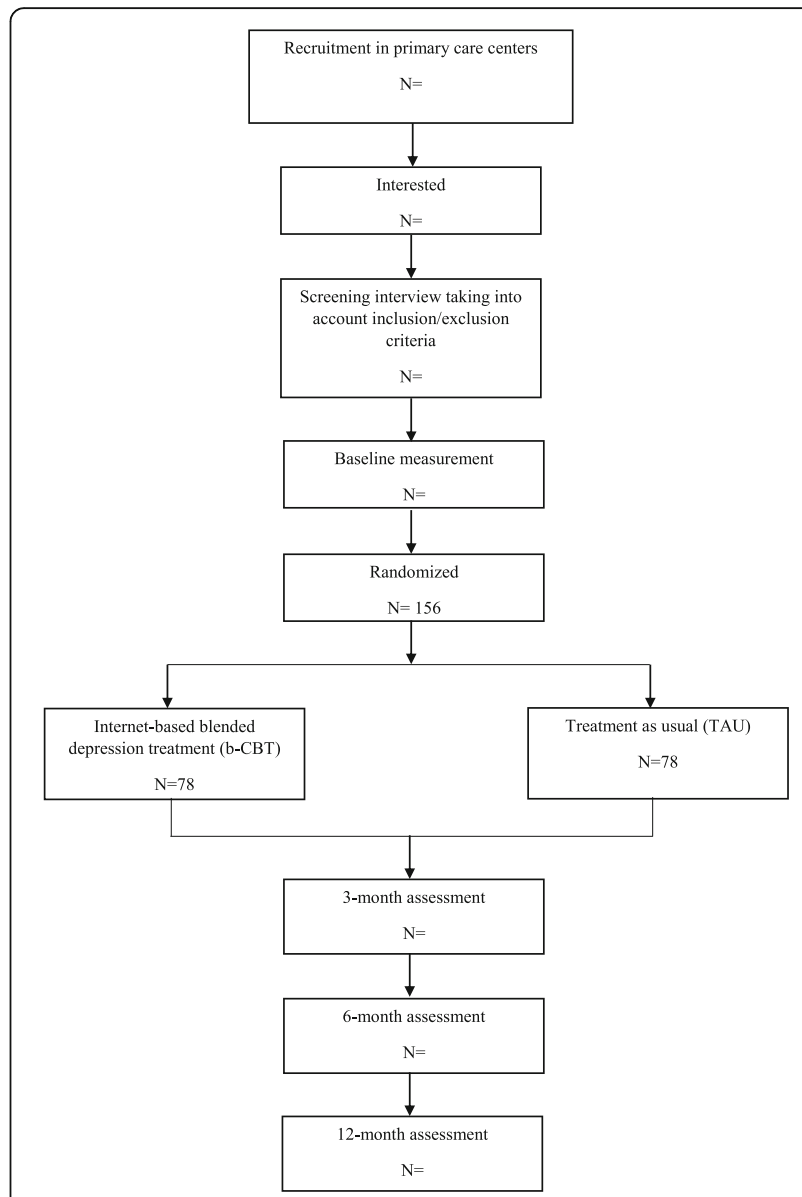

Fig. 1 Patient flow diagram

from the study at any time, without giving any reason and with no consequences. The study is registered in the United States National Institute of Health Registration System (http://www.clinicaltrials.gov) with Clinical Trials Registration Number: NCT02361684, https://clinicaltrials.gov/ct2/show/NCT02361684.

\section{Interventions \\ Blended cognitive behavioral therapy ( $b-C B T)$}

Cognitive Behavioral Therapy (CBT) is the most commonly used and recommended treatment due to its

Table 1 Inclusion/exclusion criteria

\begin{tabular}{|c|c|}
\hline Inclusion Criteria & Exclusion criteria \\
\hline Minimum age of 18 years old & $\begin{array}{l}\text { Presence of serious psychiatric comorbidities (substance dependence, bipolar affective disorder, } \\
\text { obsessive compulsive disorder, psychotic illness) }\end{array}$ \\
\hline $\begin{array}{l}\text { Meeting the DSM-IV diagnostic criteria for } \\
\text { Major Depressive Disorder }\end{array}$ & High risk for suicide \\
\hline Ability to understand and read Spanish & Receiving psychological treatment for depression at the time of recruitment \\
\hline Access to Internet and having an email address & $\begin{array}{l}\text { An increase and/or change in the pharmacological treatment (in the case of receiving it) } \\
\text { during the study period }\end{array}$ \\
\hline Providing written informed consent & Medical disease that prevents the participant from carrying out the psychological treatment \\
\hline
\end{tabular}


favorable clinical outcomes in depression [45, 46]. It usually focuses on four psychological components: 1) psycho-education, 2) behavioral activation, 3) cognitive therapy, and 4) relapse prevention. All E-COMPARED interventions must provide these four core components, and they can additionally provide two extra components.

All E-COMPARED project interventions combine individual CBT delivered through face-to-face sessions (FTF) and online sessions. The ratio used is one FTF session per three sessions delivered over the Internet. The b-CBT will be provided by therapists (clinical psychologists) who will receive special training in blended CBT and how to deliver it. Therapists will receive a manual with the corresponding procedure and content for each FTF session. All therapists will have a minimum of two years of work experience in Spanish mental health care.

Regarding the intervention protocol, this b-CBT program will be composed of three 45 -min face-to-face sessions and eight online sessions in 10 weeks.

\section{Face-to-face sessions}

The first FTF session will be held at the beginning of the treatment. It will focus on clarifying the instructions for the use of the online platform, and motivation for life changes will be addressed. In the middle of the intervention (module 6), participants will receive the second FTF session. The purpose of this session is to resolve doubts about previous therapeutic contents and reinforce commitment and adherence to treatment. The last FTF session is held at the end of the entire program. This final session is dedicated to analyzing possible difficulties and presenting and discussing relapse prevention with the participants. Between the FTF sessions, the following four online sessions will be implemented.

\section{Online treatment platform}

The online part of this intervention corresponds to the "Smiling is Fun" program [47, 48]. It is an Internetdelivered, multimedia, interactive, self-help and selfapplied program for emotional disorders (e.g., depression). It follows a transdiagnostic perspective and is based on CBT techniques. It is mainly designed to learn and practice adaptive ways to cope with depression and daily problems. It was developed within Project OPTIMI (Online Predictive Tools for Intervention in Mental Illness), funded by the VII Program Framework of the European Union. The program includes six treatment components (motivation, psycho-education, behavioral activation strategies, cognitive therapy, positive psychology strategies, and relapse prevention) addressed through eight modules. Each module and its specific objectives are shown in Table 2.

The program also includes two interactive tools that accompany the patients during the intervention: 1) The Activity diary provides feedback about mood, activities performed, and their relationship, and it also shows the mood benefits of being involved in meaningful activities; 2) The "How am I?" section offers a set of graphs and feedback to chart the user's progress in terms of level of activity, emotional discomfort, and intensity of positive and negative emotionality.

Moreover, the Internet platform includes a mobile phone component that enables daily ecological momentary assessment (EMA) of the participant's mood state (e.g., What is your mood right now?), cognitions (e.g., How much are you worrying at the moment?), activities

Table 2 Modules and objectives of "Smiling is Fun"

\begin{tabular}{|c|c|}
\hline Module & Objective \\
\hline (1) Motivation for change & $\begin{array}{l}\text { Analyze the advantages and disadvantages of changing, emphasizing the importance } \\
\text { of being motivated. }\end{array}$ \\
\hline $\begin{array}{l}\text { (2) Understanding emotional } \\
\text { problems }\end{array}$ & Recognize and understand emotional problems. \\
\hline (3) Learning to move on & $\begin{array}{l}\text { Teach the importance of "moving on" to acquire a proper level of activity and } \\
\text { involvement in life. }\end{array}$ \\
\hline (4) Learning to be flexible & $\begin{array}{l}\text { Teach a more flexible way of thinking, interpreting situations, and learning to think } \\
\text { about different alternatives. }\end{array}$ \\
\hline (5) Learning to enjoy & $\begin{array}{l}\text { Generate positive emotions, promoting the involvement in pleasant and significant } \\
\text { activities and contact with other people. }\end{array}$ \\
\hline (6) Learning to live & $\begin{array}{l}\text { Understand the importance of identifying the individual's own psychological strengths } \\
\text { and selecting and carrying out meaningful activities linked to values and goals in life. }\end{array}$ \\
\hline (7) Living and learning & Develop an action plan to boost individual psychological strengths. \\
\hline $\begin{array}{l}\text { (8) From now on, what } \\
\text { else...? }\end{array}$ & Go on and strengthen what has been learned during the program. \\
\hline
\end{tabular}


(e.g., How much did you enjoy the activities today?), social interaction (e.g., How much were you involved in social interactions today?), and sleep (e.g., How well did you sleep last night?). All the EMA measures will be time and day stamped.

\section{Treatment as usual (TAU)}

TAU is defined as the routine care delivered by the general practitioner for the treatment of depression in primary care. The type of treatment can vary depending on the GP's opinion and the severity of each patient. In Spain, the intervention generally consists of antidepressant medication. The specific type of treatment implemented will be monitored.

\section{Adherence}

If participants do not enter the online platform (for more than 15 days), they will receive emails reminding them of the importance of reviewing the modules and encouraging them to do the homework tasks. A professional platform will be used to send these emails (www.psicologiaytecnologia.com). In addition, the therapists will call the participants to schedule the FTF sessions.

\section{Measures}

The study measures and assessment times (online and by phone) are summarized in Table 3.

\section{Primary outcome measures \\ Symptoms of depression}

Patient Health Questionnaire-9 (PHQ-9) [49] will be used as a primary outcome measure. It is a 9-item questionnaire that can be used to screen and diagnose patients with depressive disorders. The nine items are each scored on a $0-3$ scale. Total scores range from 0 to 27; higher scores indicate more severe depression. The PHQ-9 has been shown to have good psychometric properties [50].

\section{Secondary outcome measures \\ Diagnostic interview}

The MINI International Neuropsychiatric Interview version 5.0 (M.I.N.I. 5.0) [40-42] will be used at screening to assess current depression and current comorbid disorders. This measure is a structured diagnostic interview based on the Diagnostic and Statistical Manual of Mental Disorders 4th edition (DSM-IV) and on International Classification of Diseases-10 (ICD-10) criteria.

Table $\mathbf{3}$ The study measures and assessment times

\begin{tabular}{|c|c|c|c|c|c|c|c|c|c|}
\hline \multirow[t]{2}{*}{ Variable } & \multirow[t]{2}{*}{ Instrument } & \multicolumn{2}{|c|}{ Screening baseline } & \multicolumn{2}{|c|}{3 months } & \multicolumn{2}{|c|}{6 months } & \multicolumn{2}{|c|}{12 months } \\
\hline & & Online & Phone & Online & $\begin{array}{l}\text { Phone } \\
\text { Pho }\end{array}$ & Online & $\overline{\text { Phone }}$ & Online & $\overline{\text { Phone }}$ \\
\hline \multicolumn{10}{|l|}{ Questions for patients } \\
\hline $\begin{array}{l}\text { Demographics and history of } \\
\text { mental health treatments }\end{array}$ & & & $x$ & & & & & & \\
\hline Diagnostic interview & M.I.N.I. & & $x$ & & & & & & $x$ \\
\hline Symptoms of depression & PHQ-9 QUIDS SR-16 & $x$ & & $x$ & & $x$ & & $x$ & \\
\hline Quality of life & EQ-5D-5 L AQol & $x$ & & $x$ & & $x$ & & $x$ & \\
\hline $\begin{array}{l}\text { Health care uptake and } \\
\text { productivity at work }\end{array}$ & TiC-P & & $x$ & & $x$ & & $x$ & & $x$ \\
\hline Treatment preference & & & $x$ & & & & & & \\
\hline $\begin{array}{l}\text { Patient expectancy of } \\
\text { treatment }\end{array}$ & CEQ & $x$ & & & & & & & \\
\hline Working alliance & WAI-SF & & & $x$ & & & & & \\
\hline Technology alliance & TAI-OT ${ }^{\mathrm{a}}$ & & & $x$ & & & & & \\
\hline Client satisfaction & CSQ & & & $x$ & & & & & \\
\hline $\begin{array}{l}\text { Satisfaction with the online } \\
\text { program }\end{array}$ & sus $^{\mathrm{a}}$ & & & $x$ & & & & & \\
\hline \multicolumn{10}{|l|}{ Questions for therapists } \\
\hline Working alliance & WAI-SF & & & $x$ & & & & & \\
\hline $\begin{array}{l}\text { Satisfaction with the online } \\
\text { program }\end{array}$ & SUS $^{\mathrm{a}}$ & & & $x$ & & & & & \\
\hline
\end{tabular}




\section{Severity of depression}

The Quick Inventory of Depressive Symptomatology-16 (QIDS SR-16) [51, 52]. This scale is a 16-item self-report that assesses the severity of depression and nine symptom domain criteria (sleep, sad mood, appetite-weight, concentration-decision making, self-view, thoughts of death or suicide, general interest, energy level, and restlessness/agitation) that define a major depressive episode according to the DSM-IV.

\section{Quality of life}

It will be measured with the EuroQol 5D (EQ-5D-5 L) [53-55] and the Assessment of Quality of Life (AQol) questionnaire [56]. The former is a self-report questionnaire that measures health-related quality of life and enables conversion to utility scores to calculate QualityAdjusted Life-Years (QALYs) [57, 58]. This scale is composed of five dimensions with 5 items related to anxiety or depression: mobility, self-care, ordinary activities, discomfort, and mood state. The AQol consists of 20 items covering five dimensions that measure the "utility" of health states: illness, independent living, social relationships, physical senses, and psychological well-being. In both instruments, the five categories are measured in a range from "no problems" to "a lot of problems".

\section{Cost measures}

\section{Health care uptake and productivity at work}

This will be evaluated with the Trimbos and iMTA Questionnaire on Costs Associated with Psychiatric Illness (TiC-P) [59]. It is an 11-item self-report questionnaire with two different parts that can be administered separately. Part I assesses the participant's health care and medication use. Part II measures lost productivity costs resulting from absenteeism (being absent from work because of illness) and presenteeism (being present at work while ill, which may lead to reduced efficiency).

\section{Other measures \\ Demographic variables and history of mental health treatments}

Personal data that include information such as age, gender, country of birth, education level, and treatments received for psychological problems.

\section{Preference for treatment}

The participants will indicate their treatment preference, choosing between the b-CBT and TAU options ("If you had the chance to choose your depression treatment, which one would you prefer to receive?").

\section{Patient expectancy of treatment}

This variable will be measured with the Credibility and Expectancy Questionnaire by Devilly and Borkovec (CEQ)
[60]. This 6-item self-report instrument measures treatment credibility and patient expectations of improvement.

\section{Therapeutic alliance}

This will be assessed using the short version of the Working Alliance Inventory (WAI-SF) [61]. This inventory is a 12-item (for patient) and 10-item (for therapist) self-report questionnaire with responses given on a 5point Likert scale ranging from 1 (never) to 5 (always), considering three dimensions: (1) therapeutic goals, (2) tasks, and (3) bond. The alliance between the patient and technologies will be assessed with the WAI Online Therapy questionnaire (TAI-OT) developed by Labpsitec (http://www.labpsitec.uji.es/esp/index.php).

\section{Client satisfaction}

The Client Satisfaction Questionnaire (CSQ) [62, 63] will be used to assess overall patient satisfaction with health and human services. It consists of 8 items measured on a 4-point scale with total scores ranging from 8 to 32 points.

\section{Satisfaction with the online program}

To assess the overall usability of the online program, the System Usability Scale (SUS) [64] will be used. It is a 10item scale, measured on a 5-point scale ranging from strongly disagree to strongly agree. Total SUS scores range from 0 to100. The questionnaire was found to be reliable and robust [65].

\section{Ecological momentary assessment (EMA) Daily functioning}

During the first and last week of treatment, twice a day (morning and evening), participants will receive a set of questions on their mobile phones about their mood state (e.g., What is your mood right now?), cognitions (e.g., How much are you worrying at the moment?), activities (e.g., How much did you enjoy activities today?), social interaction (e.g., How much were you involved in social interactions today?), and sleep (e.g., How well did you sleep last night?). They will also receive them once a week during treatment on a random day. In all cases, participants will rate their answers on a 0-10 Likert scale.

\section{Analysis}

Multiple imputation will be used to deal with missing data. The primary statistical analyses will be group comparisons of improvements in depressive symptoms. An analysis of covariance (ANCOVA) model with completers' data will be used for this purpose. Intention-totreat analyses will be used in sensitivity analyses to increase confidence in the results obtained. It is hypothesized that blended depression treatment will be equally 
as effective as care-as-usual. A non-inferiority margin and the smallest clinically acceptable difference will be considered to exist when the two-sided $95 \%$ confidence interval (the range of plausible differences between the two treatments) lies entirely above the standard mean difference of 0.20 .

\section{Discussion}

The purpose of the present study protocol is to explore the clinical effectiveness of a b-CBT for depression, compared to treatment as usual, in Spanish primary care settings. It is expected that the combination of FTF with online sessions will be at least as effective as usual care in improving depressive symptoms. Moreover, it is expected that the blended format, although somewhat more demanding in terms of commitment of clinician hours than a completely self-applied treatment, will be an adequate way to deliver empirically validated treatments, combining two key needs: the need for access and treatment dissemination and the need for therapist support during treatments. To our knowledge, this is the first study in Spain to use a b-CBT format in primary care settings, and this format could be an efficacious and cost-effective therapeutic option for the treatment of depression.

One of the strengths of the present study is the inclusion of ecological momentary assessments throughout the treatment period. This can be a relevant source of information about participants' evolution in terms of improvement and worsening and their association with specific therapeutic components. Even though these data may not be sufficient to analyze the isolated impact of each program module (given the study design implemented here), they will be a significant reflection of the real usefulness and daily impact of this intervention on the quality of life of the participants involved.

Another strength of the study is its focus on primary health care, given the need to assess the real efficacy, feasibility, and cost-effectiveness of blended treatments in these settings, with the hope that they can become a viable resource to respond to the increasing demand for psychological care.

Regarding possible limitations, we can expect some barriers and/or negative attitudes from the GPs about recommending this treatment to their patients, and high dropout rates in the treatment group can be expected (around 30\%), based on the literature [e.g., 39].

If the results of this study show the expected efficiency and efficacy of b-CBT, this could be a first step in the implementation of these treatment models in primary care as a potential solution to different problems the Healthcare system is facing at this time (such as the need to reduce costs and increasing demand from patients). In conclusion, positive results of this study could have a significant impact on primary care settings in the National Health System in Spain and in society in general.

\section{Trial status}

The study commenced recruitment in February 2015 and is currently recruiting.

\section{Abbreviations}

ANCOVA: Analysis of covariance; AQol: Assessment of Quality of Life; bCBT: Blended cognitive behavioral therapy; CBT: Cognitive Behavioral Therapy; CEQ: Credibility and Expectancy Questionnaire;

CONSORT: Consolidated Standards of Reporting Trials; CSQ: Client

Satisfaction Questionnaire; DSM-IV: Diagnostic and Statistical Manual of Mental Disorders 4 th edition; EMA: ecological momentary assessment; EQ-

5D-5 L: EuroQol 5D; FTF: Face-to-face sessions; GP: General practitioner; ICD10: International Classification of Diseases-10; M.I.N.I. 5.0: MINI International Neuropsychiatric Interview version 5.0; MDD: Major depressive disorder; PHQ9: Patient Health Questionnaire-9; QALYs: Quality-Adjusted Life-Years; QIDS SR-16: Quick Inventory of Depressive Symptomatology-16;

SPIRIT: Recommendations for Interventional Trials; SUS: System Usability Scale; TAI-OT: WAI Online Therapy; TAU: treatment as usual; TiC-P: Trimbos and iMTA Questionnaires on Costs Associated with Psychiatric IIIness; WAISF: Working Alliance Inventory; WHO: World Health Organization

\section{Acknowledgements}

This work was supported by the E-COMPARED project [European 7FP, № Agreement; 603098] under grants "PROMOSAM" [Ministerio de Economía y Competitividad; PSI2014-56303-REDT] and PROMETEOII/2013/003 program [Generalitat Valenciana]; CIBER of Physiopathology of Obesity Nutrition, an initiative of ISCIII (ISC III CB06 03/0052).

\section{Funding}

This study is funded by the European Commission FP7-Health-2013Innovation-1 program, grant agreement number: 603098.

\section{Availability of data and materials}

It is not possible to share the data because the study is still in progress. ECOMPARED data will be available upon request.

\section{Authors' contributions}

$\mathrm{RMB}, \mathrm{AGP}, \mathrm{AK}, \mathrm{HR}$ and $\mathrm{CB}$ designed the study. $\mathrm{RH}, \mathrm{EE}, \mathrm{GL}, \mathrm{BF}, \mathrm{VP}, \mathrm{PV}$ and MFM contributed significantly to the study design. MDV, RH and ME wrote the first draft of the manuscript. MDV, RH, ME and $C B$ revised the manuscript and completed the final draft. All authors contributed feedback, read, and approved the final manuscript.

\section{Ethics approval and consent to participate}

Ethical approval for this trial has been obtained for the Ethics Committee for Human Research of Spain (H1414775276823). All participants provide written informed consent before taking part in the trial.

Consent for publication

Not applicable.

\section{Competing interests}

The authors declare that they have no competing interests.

\section{Publisher's Note}

Springer Nature remains neutral with regard to jurisdictional claims in published maps and institutional affiliations.

\section{Author details}

${ }^{1}$ Department of Personality, Evaluation and Psychological Treatment, University of Valencia, Valencia, Spain. '2Department of Basic Psychology, Clinical and Psychobiology, Jaume I University, Castellón, Spain. ${ }^{3}$ Department of Personality, Evaluation and Psychological Treatment, University of Zaragoza, Zaragoza, Spain. ${ }^{4}$ CIBER Fisiopatología Obesidad y Nutrición (CIBEROBN), Instituto Carlos III, Madrid, Spain. ${ }^{5}$ Hospital Universitario de la 
Ribera. Alzira (Valencia), Valencia, Spain. ${ }^{6}$ Complejo Asistencial de Zamora, Zamora, Spain. ${ }^{7}$ Section Clinical Psychology, Vrije Universiteit Amsterdam and $\mathrm{EMGO}+$ Institute for Health Care and Research, Amsterdam, the Netherlands. ${ }^{8}$ Department of Psychiatry, VU University Medical Centre and EMGO+ Institute for Health Care and Research, Amsterdam, the Netherlands.

Received: 27 October 2017 Accepted: 22 February 2018

Published online: 23 March 2018

\section{References}

1. Lépine J-P, Briley M. The increasing burden of depression. Neuropsychiatr Dis Treat. 2011;7(Suppl 1):3-7.

2. de Graaf R, ten Have M, van Gool C, van Dorsselaer S. Prevalence of mental disorders and trends from 1996 to 2009. Results from the Netherlands mental health survey and incidence study-2. Soc Psychiatry Psychiatr Epidemiol. 2012; https://doi.org/10.1007/s00127-010-0334-8.

3. Kessler RC, Chiu WT, Demler O, Walters EE, Walters EE. Prevalence, severity, and comorbidity of 12-month DSM-IV disorders in the national comorbidity survey replication. Arch Gen Psychiatry. 2005; https://doi.org/10.1001/ archpsyc.62.6.617.

4. Alonso J, Lépine J-P. ESEMeD/MHEDEA 2000 scientific committee. Overview of key data from the European study of the epidemiology of mental disorders (ESEMeD). J Clin Psychiatry. 2007;68(Suppl 2):3-9.

5. Mathers CD, Loncar D. Projections of global mortality and burden of disease from 2002 to 2030. PLoS Med. 2006; https://doi.org/10.1371/journal.pmed. 0030442.

6. World Health Association. The global burden of disease: 2004 update. Geneva: WHO; 2004

7. Huhn M, Tardy M, Spineli LM, Kissling W, Förstl H, Pitschel-Walz G, et al. Efficacy of pharmacotherapy and psychotherapy for adult psychiatric disorders. JAMA Psychiatry. 2014; https://doi.org/10.1001/jamapsychiatry. 2014.112.

8. Cuijpers P, van Straten A, van Oppen P, Andersson G. Are psychological and pharmacologic interventions equally effective in the treatment of adult depressive disorders? A meta-analysis of comparative studies. J Clin Psychiatry. 2008;69(11):1675-85.

9. Cuijpers P, Sijbrandij M, Koole SL, Andersson G, Beekman AT, Reynolds CF. The efficacy of psychotherapy and pharmacotherapy in treating depressive and anxiety disorders: a meta-analysis of direct comparisons. World Psychiatry. 2013; https://doi.org/10.1002/wps.20038.

10. Kazdin AE, Blase SL. Rebooting psychotherapy research and practice to reduce the burden of mental illness. Perspect Psychol Sci. 2011; https://doi. org/10.1177/1745691610393527.

11. Aragonès E, Piñol JL, Labad A, Masdéu RM, Pino M, Cervera J. Prevalence and determinants of depressive disorders in primary care practice in Spain Int J Psychiatry. 2004; https://doi.org/10.2190/C25N-W4NY-BN8W-TXN2.

12. King M, Nazareth I, Levy G, Walker C, Morris R, Weich S, et al. Prevalence of common mental disorders in general practice attendees across Europe. $\mathrm{Br}$ J Psychiatry. 2008; https://doi.org/10.1192/bjp.bp.107.039966.

13. Serrano-Blanco A, Palao DJ, Luciano JV, Pinto-Meza A, Luján L, Fernández A, et al. Prevalence of mental disorders in primary care: results from the diagnosis and treatment of mental disorders in primary care study (DASMAP). Soc Psychiatry Psychiatr Epidemiol. 2010; https://doi.org/10.1007/ s00127-009-0056.

14. Aragonès E, Piñol JL, Labad A, Folch S, Mèlich N. Detection and management of depressive disorders in primary care in Spain. Int J Psychiatry Med. 2004; https://doi.org/10.2190/N835-FDYX-2E2E-V8XM.

15. Gabilondo A, Rojas-Farreras S, Rodráguez A, Ferníndez A, Pinto-Meza A, Vilagut $\mathrm{G}$, et al. Use of primary and specialized mental health care for a major depressive episode in Spain by ESEMeD respondents. Psychiatr Serv. 2011; https://doi.org/10.1176/ps.62.2.pss6202_0152.

16. Fairburn CG, Patel V. The impact of digital technology on psychological treatments and their dissemination. Behav Res Ther. 2017; https://doi.org/10. 1016/j.brat.2016.08.012

17. Andrews G, Cuijpers P, Craske MG, McEvoy P, Titov N. Computer therapy for the anxiety and depressive disorders is effective, acceptable and practical health care: a meta-analysis. PLoS One. 2010; https://doi.org/10.1371/journal. pone.0013196.

18. Richards D, Richardson T. Computer-based psychological treatments for depression: a systematic review and meta-analysis. Clin Psychol Rev. 2012; https://doi.org/10.1016/j.cpr.2012.02.004.
19. Andersson G, Cuijpers P. Internet-based and other computerized psychological treatments for adult depression: a meta-analysis. Cogn Behav Ther. 2009; https://doi.org/10.1080/16506070903318960.

20. Baumeister $H$, Reichler $L$, Munzinger $M$, Lin J. The impact of guidance on internet-based mental health interventions - a systematic review. Internet Interv. 2014; https://doi.org/10.1016/j.invent.2014.08.003.

21. Andersson G. Internet-delivered psychological treatments. Annu Rev Clin Psychol. 2016; https://doi.org/10.1146/annurev-clinpsy-021815-093006.

22. Johansson R, Andersson G. Internet-based psychological treatments for depression. Expert Rev Neurother. 2012; https://doi.org/10.1586/ern.12.63.

23. Cuijpers P, Donker T, van Straten A, Li J, Andersson G. Is guided self-help as effective as face-to-face psychotherapy for depression and anxiety disorders?. A systematic review and meta-analysis of comparative outcome studies. Psychol Med. 2010; https://doi.org/10.1017/S0033291710000772.

24. Andersson $G$, Hesser $H$, Veilord A, Svedling L, Andersson F, Sleman O, et al. Randomised controlled non-inferiority trial with 3-year follow-up of internetdelivered versus face-to-face group cognitive behavioural therapy for depression. J Affect Disord. 2013; https://doi.org/10.1016/j.jad.2013.08.022.

25. Andersson G, Cuijpers P, Carlbring P, Riper H, Hedman E. Guided internetbased vs. face-to-face cognitive behavior therapy for psychiatric and somatic disorders: a systematic review and meta-analysis. World Psychiatry. 2014; https://doi.org/10.1002/wps.20151.

26. Wentzel J, van der Vaart R, Bohlmeijer ET, van Gemert-Pijnen JEWC. Mixing online and face-to-face therapy: how to benefit from blended care in mental health care. JMIR Ment Heal. 2016; https://doi.org/10. 2196/mental.4534.

27. Månsson KNT, Skagius Ruiz E, Gervind E, Dahlin M, Andersson G. Development and Initial evaluation of an internet-based support system for face-to-face cognitive behavior therapy: a proof of concept study. J Med Internet Res. 2013; https://doi.org/10.2196/jmir.3031.

28. Wright JH, Wright AS, Albano AM, Basco MR, Goldsmith LJ, Raffield T, et al. Computer-assisted cognitive therapy for depression: maintaining efficacy while reducing therapist time. Am J Psychiatry. 2005; https://doi.org/10. 1176/appi.ajp.162.6.1158.

29. Clough BA, Casey LM. Technological adjuncts to increase adherence to therapy: a review. Clin Psychol Rev. 2011; https://doi.org/10.1016/j.cpr. 2011.03.006.

30. Ebert D, Tarnowski T, Gollwitzer M, Sieland B, Berking M. A transdiagnostic internet-based maintenance treatment enhances the stability of outcome after inpatient cognitive behavioral therapy: a randomized controlled trial. Psychother Psychosom. 2013; https://doi. org/10.1159/000345967.

31. Holländare $F$, Johnsson $S$, Randestad M, Tillfors M, Carlbring $P$, Andersson $G$, et al. Randomized trial of internet-based relapse prevention for partially remitted depression. Acta Psychiatr Scand. 2011; https://doi.org/10.1111/j.1600-0447.2011.01698.x.

32. Craske MG, Rose RD, Lang A, Welch SS, Campbell-Sills L, Sullivan G, et al. Computer-assisted delivery of cognitive behavioral therapy for anxiety disorders in primary-care settings. Depress Anxiety. 2009; https://doi.org/10.1002/da.20542.

33. Moher D, Schulz KF, Altman DG, CONSORT Group (Consolidated standards of reporting trials). The CONSORT statement: revised recommendations for improving the quality of reports of parallel-group randomized trials. J Am Podiatr Med Assoc. 2001;91(8):437-42.

34. Moher D, Hopewell S, Schulz KF, Montori V, Gøtzsche PC, Devereaux PJ, et al. CONSORT 2010 explanation and elaboration: updated guidelines for reporting parallel group randomised trials. J Clin Epidemiol. 2010; https:// doi.org/10.1016/j.jclinepi.2010.03.004

35. Eysenbach G, CONSORT-EHEALTH Group C-E. CONSORT-EHEALTH: Improving and standardizing evaluation reports of web-based and mobile health interventions. J Med Internet Res. 2011; https://doi.org/10.2196/jmir.1923.

36. Chan A-W, Tetzlaff JM, Altman DG, Laupacis A, Gøtzsche PC, Krleža-Jerić K, et al. SPIRIT 2013 statement: defining standard protocol items for clinical trials. Ann Intern Med. 2013; https://doi.org/10.7326/0003-4819-158-3201302050-00583.

37. Chan A-W, Tetzlaff JM, Gøtzsche PC, Altman DG, Mann H, Berlin JA, et al. SPIRIT 2013 explanation and elaboration: guidance for protocols of clinical trials. BMJ. 2013; https://doi.org/10.1136/bmj.e7586.

38. Cohen J. Statistical power analysis for the behavioral sciences. Second. New York: Lawrence Erlbaum Associates; 1988. 
39. van Ballegooijen W, Cuijpers P, van Straten A, Karyotaki E, Andersson G, Smit $J H$, et al. Adherence to internet-based and face-to-face cognitive behavioural therapy for depression: a meta-analysis. PLoS One. 2014; https://doi.org/10.1371/journal.pone.0100674.

40. Sheehan DV, Lecrubier Y, Sheehan KH, Amorim P, Janavs J, Weiller E, et al. The Mini-International Neuropsychiatric Interview (M.I.N.I.): the development and validation of a structured diagnostic psychiatric interview for DSM-IV and ICD-10. J Clin Psychiatry. 1998;59(Suppl 20):22. 33-57

41. Lecrubier Y, Sheehan D, Weiller E, Amorim P, Bonora I, Harnett Sheehan K, et al. The MINI international neuropsychiatric interview (MINI). A short diagnostic structured interview: reliability and validity according to the CIDI. Eur Psychiatry. 1997; https://doi.org/10.1016/S0924-9338(97)83296-8.

42. Ferrando L, Bobes J, Gibert J, Soto M, Soto O. MINI. In: Entrevista Neuropsiquiátrica Internacional Versión en Español 5.0.0. Madrid: DSM-IV; 2000.

43. World Medical Association. World medical association declaration of Helsinki: ethical principles for medical research involving human subjects. JAMA. 2013; https://doi.org/10.1001/jama.2013.281053.

44. Food Drug Administration. International conference on harmonization, good clinical practice: consolidated guidelines. Fed Regist. 1997;62 25692-709

45. Cuijpers $P$, van Straten A, Warmerdam L. Behavioral activation treatments of depression: a meta-analysis. Clin Psychol Rev. 2007; https://doi.org/10.1016/j.cpr.2006.11.001

46. Mazzucchelli T, Kane R, Rees C. Behavioral activation treatments for depression in adults: a meta-analysis and review. Clin Psychol Sci Pract. 2009; https://doi.org/10.1111/j.1468-2850.2009.01178.x.

47. Botella C, Mira A, Garcia-Palacios A, Quero S, Navarro MV, Riera López del Amo A, et al. Smiling is fun: a coping with stress and emotion regulation program. Stud Health Technol Inform. 2012;181:123-7.

48. Botella Arbona C, Mira A, Herrero R, García Palacios A, Baños Rivera RM. Un programa de intervención auto-aplicado a través de Internet para el tratamiento de la depresión: "Sonreír es divertido". Aloma Rev Psico ciències l'educació i l'esport. 2015;33(33):39-48.

49. Kroenke K, Spitzer RL, Williams JB. The PHQ-9: validity of a brief depression severity measure. J Gen Intern Med. 2001;16(9):606-13.

50. Wittkampf KA, Naeije L, Schene AH, Huyser J, van Weert HC. Diagnostic accuracy of the mood module of the patient health questionnaire: a systematic review. Gen Hosp Psychiatry. 2007; https://doi.org/10.1016/j. genhosppsych.2007.06.004.

51. Rush AJ, Trivedi MH, Ibrahim HM, Carmody TJ, Arnow B, Klein DN, et al. The 16-item quick inventory of depressive symptomatology (QIDS), clinician rating (QIDS-C), and self-report (QIDS-SR): a psychometric evaluation in patients with chronic major depression. Biol Psychiatry. 2003:54(5):573-83.

52. Trujols J, De Diego-Adeliño J, Feliu-Soler A, Iraurgi I, Puigdemont D, Álvarez E. et al., The Spanish version of the quick inventory of depressive symptomatology-self-report (QIDS-SR16): a psychometric analysis in a clinical sample. J Affect Disord. 2014; https://doi.org/10. 1016/j.jad.2014.08.011.

53. EuroQol Group. EuroQol-a new facility for the measurement of healthrelated quality of life. Health Policy. 1990;16(3):199-208.

54. Herdman M, Gudex C, Lloyd A, Janssen M, Kind P, Parkin D, et al. Development and preliminary testing of the new five-level version of EQ-5D (EQ-5D-5L). Qual Life Res. 2011; https://doi.org/10.1007/s11136-011-9903-X.

55. Badia X, Roset M, Montserrat S, Herdman M, Segura A. The Spanish version of EuroQol: a description and its applications. European quality of life scale. Med Clin. 1999;112(Suppl 1):79-85.

56. Hawthorne G, Richardson J, Osborne R. The assessment of quality of life (AQoL) instrument: a psychometric measure of health-related quality of life. Qual Life Res. 1999;8(3):209-24.

57. Keeley $\mathrm{T}, \mathrm{Al}$-Janabi H, Lorgelly $\mathrm{P}, \mathrm{Coast} J$. A qualitative assessment of the content validity of the ICECAP-A and EQ-5D-5L and their appropriateness for use in health research. PLoS One. 2013; https://doi.org/10.1371/journal. pone.0085287.

58. Janssen MF, Pickard AS, Golicki D, Gudex C, Niewada M, Scalone L, et al. Measurement properties of the EQ-5D-5L compared to the EQ5D-3L across eight patient groups: a multi-country study. Qual Life Res. 2013; https://doi.org/10.1007/s11136-012-0322-4.

59. Hakkaart-van Roijen L, van Straten A, Tiemens B, Donker M. Manual Trimbos/iMTA questionnaire for costs associated with psychiatric illness
(TiC-P). Rotterdam: Institute for Medical Technology Assessment, Erasmus University Rotterdam; 2002.

60. Devilly GJ, Borkovec TD. Psychometric properties of the credibility/ expectancy questionnaire. J Behav Ther Exp Psychiatry. 2000;31(2):73-86.

61. Hatcher RL, Gillaspy JA. Development and validation of a revised short version of the working alliance inventory. Psychother Res. 2006; https://doi. org/10.1080/10503300500352500.

62. Larsen DL, Attkisson CC, Hargreaves WA, Nguyen TD. Assessment of client/ patient satisfaction: development of a general scale. Eval Program Plann. 1979; https://doi.org/10.1016/0149-7189(79)90094-6.

63. Attkisson C, Greenfield TK. The client satisfaction questionnaire (CSQ) scales and the service satisfaction Scale-30. Outcomes assess. Clin Pract. 1996:30:120-7.

64. Brooke J. SUS: a quick and dirty usability scale. Usability Eval Ind. 1996; 189:4-7.

65. Bangor A, Kortum PT, Miller JT. An empirical evaluation of the system usability scale. Int J Hum Comput Interact. 2008; https://doi.org/10.1080/ 10447310802205776

\section{Submit your next manuscript to BioMed Central and we will help you at every step:}

- We accept pre-submission inquiries

- Our selector tool helps you to find the most relevant journal

- We provide round the clock customer support

- Convenient online submission

- Thorough peer review

- Inclusion in PubMed and all major indexing services

- Maximum visibility for your research

Submit your manuscript at www.biomedcentral.com/submit
Biomed Central 